\title{
N6-methyladenosine-mediated SH3BP5-AS1 upregulation promotes GEM chemoresistance in pancreatic cancer by competitively sponging miR- 139-5p and activating the Wnt signaling pathway
}

Chengjie Lin ( $\sim$ linchengjie8@aliyun.com )

the first affiliated hospital of fujian medical university

Yan Wang

Fujian Medical University

Yihong Dong

Fujian Medical University

Shihui Lai

Fujian Medical University

Liang Wang

Fujian Medical University

Xiang Zhang

Fujian Medical University

Shangeng Weng

Fujian Medical University

\section{Research Article}

Keywords: SH3BP5-AS1, N6-methyladenosine, pancreatic cancer, chemoresistance

Posted Date: January 17th, 2022

DOI: https://doi.org/10.21203/rs.3.rs-1242747/v1

License: (c) (1) This work is licensed under a Creative Commons Attribution 4.0 International License. Read Full License 


\section{Abstract \\ Background}

Pancreatic cancer (PC) is highly malignant. Chemotherapy is the main treatment strategy, especially for patients with advanced PC. However, chemoresistance has always been a frequently encountered bottleneck. Hence, there is an urgent need to enhance the sensitivity of PC to gemcitabine (GEM).

\section{Methods}

A patient-derived xenograft model was used for screening the hub IncRNAs affecting GEM chemoresistance. Transwell and colony formation assays were used to assess the migration, invasion, and stemness of PC cells. qRT-PCR and western blot analyses were performed to detect the mRNA and protein levels indicated in this study, respectively. The MTT assay was performed to assess the viability of PC cells in vitro. MeRIP-qPCR was used to determine the N6-methyladenosine (m6A) modification level. RIP and pull-down were used to investigate the interaction between SH3BP5-AS1 and miR-139-5p, as well as the interactions among SH3BP5-AS1, ALKBH5, and IGF2BP1. The functional mechanism of SH3BP5-AS1 in PC was identified by gain- and loss-of-function assays and high-throughput sequencing in vitro.

\section{Results}

We demonstrated that SH3BP5-AS1 was significantly upregulated in GEM-resistant PC and predicted a poorer prognosis. SH3BP5-AS1 stability was regulated by ALKBH5/IGF2BP1-mediated m6A modification. Loss of SH3BP5-AS1 reduced PC cell migration and invasion and enhanced the sensitivity of PC to GEM, as confirmed by gain- and loss-of-function assays in vitro and in vivo. Bioinformatics analysis revealed that SH3BP5-AS1 acted as a ceRNA against miR-139-5p and directly targeted CTBP1, affecting the biological behavior of PC cells. The mechanistic studies revealed that the upregulation of SH3BP5-AS1 increased CTBP1 expression by directly activating the Wnt signaling pathway, promoting GEM resistance.

\section{Conclusion}

This study revealed that SH3BP5-AS1 activated Wnt signaling pathway by sponging miR-139-5p, upregulating CTBP1 expression, and contributing to the sensitivity of PC cells to GEM. SH3BP5-AS1 might be a potential target for PC therapy.

\section{Background}

Pancreatic cancer (PC) is highly malignant and is characterized by high recurrence rates and low resection rates. Its 5 -year survival rate is only $9 \%$ [1]. The incidence rate of PC is increasing yearly. In 
2018 , the number of newly diagnosed cases of PC worldwide was similar to the number of deaths in the same year [2]. Currently, surgical resection combined with adjuvant chemotherapy is the standard treatment. Chemotherapy is the main treatment, especially for patients with advanced PC. Gemcitabine (GEM) is a first-line chemotherapy drug approved for the treatment of advanced PC, either alone or in combination with other chemotherapeutic agents [3]. Although in recent clinical trial combinations of GEM with other drugs resulted in relative improvements in median survival time, PC cells are less sensitive to GEM, resulting in unsatisfactory chemotherapy effects, owing to natural and/or acquired resistance to GEM [4]. Hence, there is an urgent need to enhance the sensitivity of PC to GEM. Therefore, it is important to explore the mechanisms underlying chemoresistance in PC and to optimize GEM formulations and medication regimens.

Long non-coding RNA (IncRNA) has been found to be involved in tumor chemoresistance in different pathways. LncRNAs PVT1, GSTM3TV2, and LINC00346 have been confirmed to promote GEM resistance in PC by competitively binding downstream genes [5-7]. All these studies indicated that IncRNAs were closely related to the chemoresistance of tumors. In this study, a patient-derived xenograft (PDX) model was used and genome sequencing was conducted. We found that the expression of SH3BP5-AS1 was positively correlated to the GEM resistance of PC, indicating an important role of SH3BP5-AS1 in the resistance of PC to GEM. Lina et al. found that the expression of SH3BP5-AS1 was upregulated in recurrent neuroblastoma and head and neck cancer [8, 9]. At present, few reports on SH3BP5-AS1 are available, and the role of SH3BP5-AS1 in PC, including the causes for its abnormal expression in PC and the mechanism by which it affects the sensitivity to GEM, remains to be elucidated.

The N6-methyladenosine (m6A) modification has been confirmed to affect RNA stability, thereby affecting gene expression. In recent years, a large number of studies on m6A modifications of IncRNAs regulating tumor activity have been published. The m6A modification mainly occurs on the adenine residue in the RRACH sequence. Its functions are mainly characterized as "writer," "eraser," and "reader." First, IncRNA is modified by a methylase "writer" (METTL3/14, WTAP, etc.) or a demethylase "eraser" (ALKBH5/FTO). Then it is recognized by a "reader" (YTHDF1/2/3, IGF2BP1, etc.) for stabilization or degradation[10]. In this study, we found that the expression level of IncRNA SH3BP5-AS1 is regulated by m6A modification, and its abnormal expression affects the sensitivity of PC to GEM. We further explored the potential effects of SH3BP5-AS1 on GEM resistance of PC and attempted to reduce GEM resistance of PC cells by regulating SH3BP5-AS1. We provide an experimental basis for the development of targeted drugs that enhance GEM sensitivity.

\section{Methods}

\section{Clinical specimens}

In total, 87 PC samples were collected from the First Affiliated Hospital of Fujian Medical University between 2008 and 2018. The clinicopathological characteristics including age, sex, alcohol history, smoking history, CEA, CA199, tumor size, tumor stage, lymph node invasion, and metastasis were 
recorded. This study was approved by the Ethics Committee of the First Affiliated Hospital of Fujian Medical University (Fujian, China).

\section{Cell culture}

The human PC cell lines AsPC-1, CFPAC-1, Capan-1, SW1990, PANC-1, SW1990, BxPC-3, and MIA-PaCa-2 and the non-cancerous HPDE6C7 cell line were purchased from the American Type Culture Collection (ATCC; Manassas, VA, USA). Cells were cultured in Dulbecco's modified Eagle's medium (DMEM) supplemented with $10 \%$ fetal bovine serum (FBS) and $1 \%$ antibiotic/antifungal solution (Biowest, Nuaillé, France) in $5 \% \mathrm{CO}_{2}$ at $37^{\circ} \mathrm{C}$ in a humidified atmosphere.

\section{Immunohistochemistry}

Tissues were fixed with $10 \%$ formalin for about $48 \mathrm{~h}$ and paraffin-embedded for immunohistochemistry (IHC) assays. The protocol was previously described [10]. All antibodies and related reagents are shown in Table S1.

\section{Western blot}

Proteins were extracted from homogenized tissues or cultured cells, sample buffer was added, samples were heated, and proteins were separated by SDS-PAGE and transferred to a polyvinylidene fluoride membrane (Bio-Rad Laboratories, Hercules, CA, USA). The membrane was blocked in 5\% skim milk for about $1 \mathrm{~h}$ and incubated with primary antibody (Table S1) at $4^{\circ} \mathrm{C}$ overnight. Then the membrane was washed and incubated with secondary antibody for $2 \mathrm{~h}$, and after washing, protein bands were visualized using enhanced chemiluminescence (ECL) substrate and photographed. Relative expression levels of proteins were quantified by ImageJ software version 1.8.0 (National Institutes of Health, Bethesda, MD, USA).

\section{Quantitative reverse-transcriptase PCR}

Total RNA was isolated using TRIzol reagent (Invitrogen, Carlsbad, CA, USA) following the manufacturer's instructions, and cDNA was synthesized with random primers (Table S2) using a reverse transcription kit (TOYOBO, Japan) following the manufacturer's instructions. qRT-PCR was performed using Fast Start Universal SYBR Green Master Mix (Roche Diagnostics GmbH, Mannheim, Germany).

\section{Colony formation assay}

Cells were cultured in 6-well plates (500 cells/well) for 14 days. Then, the colonies were fixed by methanol/acetic acid (3:1, v/v) and stained with $0.5 \%$ crystal violet (Sigma-Aldrich, St. Louis, MO, USA). The colonies were counted using a microscope (Olympus IX81).

\section{RNA immunoprecipitation assay}

The RNA immunoprecipitation (RIP) assay was performed using a Magna RIP ${ }^{\mathrm{TM}}$ RNA-Binding Protein Immunoprecipitation Kit (Millipore, USA). Briefly, cell lysates were immunoprecipitated with beads conjugated with anti-AGO2 or anti-IgG at $4^{\circ} \mathrm{C}$ for $6 \mathrm{~h}$. Then, the immunoprecipitates were treated with 
$0.1 \% \mathrm{SDS} /$ Proteinase $\mathrm{K}\left(0.5 \mathrm{mg} / \mathrm{mL}, 30 \mathrm{~min}\right.$ at $\left.55^{\circ} \mathrm{C}\right)$, and immunoprecipitated RNAs were detected by qRT-PCR.

\section{Methylated RNA immunoprecipitation qPCR}

An miRNeasy kit (\#217004, QIAGEN) was used for isolating the total RNA fraction. The GenElute mRNA Miniprep kit (MRN10, Sigma-Aldrich) was used for extracting mRNA from the total RNA fraction. Then, a Magna MeRIP m6A kit (\#17-10499, Millipore) was used according to the manufacturer's instructions, and m6A enrichment was analyzed by qPCR.

\section{RNA pull-down assay}

A Pierce Magnetic RNA-Protein Pull-Down Kit (Thermo Fisher Scientific, 20164) was used for the RNA pull-down assay. Briefly, cell extracts were treated with RNase-free DNase I and incubated with biotinylated SH3BP5-AS1 in the presence of streptavidin magnetic beads, which can capture the proteins/miRNAs potentially interacting with SH3BP5-AS1. A Pierce ${ }^{\mathrm{TM}}$ RNA 3' End Desthiobiotinylation Kit (Thermo, 20163) was used for SH3BP5-AS1 biotinylation labeling. Proteins and RNAs in the captured protein-RNA complex were analyzed using western blot and RT-qPCR, respectively.

\section{Drug sensitivity assay}

To assess the chemosensitivity of PC cells to GEM, cells were treated with different concentrations of $\operatorname{GEM}(0.1,1,10$, and $100 \mu \mathrm{M})$ for $72 \mathrm{~h}$ in 96-well plates as previously described [7]. An MTT kit was used to detect the number of viable cells, and the half maximal inhibitory concentration $\left(\mathrm{IC}_{50}\right)$ was calculated to assess drug sensitivity.

\section{Overexpression plasmid and siRNA transfection}

The overexpression plasmid and small interfering RNA (siRNA) were purchased from GenePharma (Shanghai, China). Cells at a concentration of $5 \times 10^{5}$ cells $/ \mathrm{ml}$ per well were cultured in 6 -well plates for 2 days. PC cells were transfected with overexpression plasmid or negative controls using Lipofectamine 3000 (Invitrogen) at a final concentration of $50 \mathrm{nM}$. Cells were transfected with siRNAs or si-NCs using Lipofectamine 3000 (Invitrogen) at a final concentration of $100 \mathrm{nM}$.

\section{Cell migration and invasion assay}

The Transwell assay was used to analyze cell migration and invasion. Chambers coated with Matrigel (Corning Labware Products Inc., Corning, NY, USA) were used for invasion assays, and uncoated chambers with Matrigel were used for cell migration assays. First, $500 \mu$ l basal medium (without serum) was added into the chambers. After incubation for $2 \mathrm{~h}$, the chambers were placed in a 24-well plate containing cell culture medium with $10 \%$ FBS. Then, $100 \mu$ l cell suspension $\left(2 \times 10^{5}\right.$ cells $\left./ \mathrm{ml}\right)$ was added into the chamber. After $24 \mathrm{~h}$, the chambers were fixed with paraformaldehyde and then stained with crystal violet to allow visualization. Cells were photographed under an Olympus IX73 microscope (Olympus).

\section{In vivo assay}


Six-week-old male BALB/c nude mice were purchased from the Shanghai Laboratory Animal Center for in vivo experiments. Cells stably transfected with the sh-SH3BP5-AS1 lentivirus or empty vectors were injected subcutaneously or into the tail vein of the selected mice. After 4 weeks, the tumors were harvested and their weight and volume were measured. The experimental protocols were approved by the Fujian Medical University Ethics Review Committee.

\section{Statistical analysis}

GraphPad software v.6 and SPSS software version 21.0 (IBM Corp., Armonk, NY, USA) were used for statistical analysis. Measured data are reported as the mean \pm standard deviation (SD). Student's $t$-test or one-way ANOVA was used to evaluate means between groups. Pearson correlation analysis was performed to determine correlations. Survival was analyzed by Kaplan-Meier curves. The correlations between expression levels and clinicopathological features were analyzed using either the $\chi^{2}$ test or Fisher's exact test. Data are reported from at least three independent experiments. $P<0.05$ was considered to be statistically significant.

\section{Results}

\section{SH3BP5-AS1 was upregulated in GEM-treated PDX pancreatic cancer}

To explore the mechanism of GEM resistance in PC, we first established a PDX model, which was treated with either saline or GEM (Fig. 1A). We performed genomic sequencing of p4-pdx treated with GEM or the negative control to screen for hub genes. The top 10 differentially expressed IncRNAs are shown in Fig. 1B. The role of SH3BP5-AS1, one of the most strongly upregulated IncRNAs, in PC tumorigenesis and chemosensitivity was further investigated. The TCGA database suggested that SH3BP5-AS1 is closely related to the tumor grade of PC (Fig. 1C). Analysis of the correlation between SH3BP5 expression and clinicopathological data revealed that high SH3BP5-AS1 expression was closely related to tumor TNM stage, tumor size, and degree of differentiation in our center (Table 1). SH3BP5-AS1 expression was measured in 87 pair-matched PC tumor and adjacent normal tissues by RT-qPCR, and the results suggested that SH3BP5-AS1 was significantly upregulated in tumor tissue compared to normal tissue (Fig. 1D). ROC curve analysis revealed that it was an independent risk factor for the prognosis of PC (Fig. 1E). The survival of patients with low SH3BP5-AS1 expression was significantly better than that of patients with high SH3BP5-AS1 expression (Fig. 1F). At the same time, the expression levels of SH3BP5AS1 in five PC cell lines and normal pancreatic cells were detected in vitro. SH3BP5-AS1 expression was significantly higher in PC cells than in normal pancreatic cells (Fig. 1G). Collectively, the above results demonstrated that SH3BP5-AS1 plays a key role in PC tumorigenicity and might serve as a key regulator of GEM resistance. 
Table 1

Relationship between SH3BP5-AS1 expression and clinicopathological parameters in $87 \mathrm{PC}$ patients.

\begin{tabular}{|c|c|c|c|c|}
\hline \multirow[t]{2}{*}{ Variables } & \multirow[t]{2}{*}{ All cases } & \multicolumn{2}{|c|}{ SH3BP5-AS1 expression } & \multirow[t]{2}{*}{$P$} \\
\hline & & $\operatorname{Low}(n=31)$ & High $(n=56)$ & \\
\hline \multicolumn{5}{|c|}{ Age (years) } \\
\hline$<50$ & 40 & 14 & 26 & 0.910 \\
\hline$\geq 50$ & 47 & 17 & 30 & \\
\hline \multicolumn{5}{|l|}{ Gender } \\
\hline Male & 59 & 22 & 37 & 0.640 \\
\hline Female & 28 & 9 & 19 & \\
\hline \multicolumn{5}{|c|}{ Alcohol history } \\
\hline No & 41 & 14 & 27 & 0.785 \\
\hline Yes & 46 & 17 & 29 & \\
\hline \multicolumn{5}{|l|}{ Smoking } \\
\hline No & 37 & 12 & 25 & 0.592 \\
\hline Yes & 50 & 19 & 31 & \\
\hline \multicolumn{5}{|l|}{ CEA } \\
\hline normal & 17 & 9 & 8 & 0.097 \\
\hline High & 70 & 22 & 48 & \\
\hline \multicolumn{5}{|l|}{ CA199 } \\
\hline normal & 26 & 12 & 14 & 0.181 \\
\hline High & 61 & 19 & 42 & \\
\hline \multicolumn{5}{|c|}{ Tumor size (cm) } \\
\hline$<4$ & 52 & 23 & 29 & $0.041^{*}$ \\
\hline$\geq 4$ & 35 & 8 & 27 & \\
\hline \multicolumn{5}{|c|}{ TNM stage } \\
\hline$|-| \mid$ & 35 & 16 & 19 & 0.02 \\
\hline III-IV & 45 & 32 & 13 & \\
\hline
\end{tabular}




\begin{tabular}{|lllll|}
\hline Variables & All cases & \multicolumn{2}{l}{ SH3BP5-AS1 expression } & $P$ \\
\cline { 3 - 4 } & & Low $(\boldsymbol{n = 3 1 )})$ & High $(\boldsymbol{n = 5 6 )}$ & \\
\hline Lymph node invasion & & & 19 & $0.035^{*}$ \\
\hline Absent & 36 & 17 & 13 & \\
\hline Present & 44 & 31 & & 0.034 \\
\hline Metastasis & & & 29 & \\
\hline No & 63 & 34 & 3 & \\
\hline Yes & 17 & 14 & & \\
\hline *P $<0.05$. & & & & \\
\hline
\end{tabular}

Abnormal expression of SH3BP5-AS1 was involved in the invasion, migration, and stemness of pancreatic cancer cells

To further explore the effects of SH3BP5-AS1 on the biological behavior of PC cells, PC cells (BxPC3, MIA Paca-2, and PANC-1) were transfected with lentiviral vectors encoding SH3BP5-AS1 inserts or short hairpin RNA (shRNA) and Transwell and colony formation assays were conducted. After silencing SH3BP5-AS1, the invasion, migration, and colony formation abilities of PC cells (BxPC3, MIA Paca-2) were significantly reduced (Fig. 2A-D and F-I). On the contrary, overexpression of SH3BP5-AS1 enhanced the above cellular behaviors (Fig. S2A-E).

Epithelial-to-mesenchymal transition (EMT) has been proved to be closely related to cell invasion and migration[11]. Therefore, we also detected EMT-related proteins. The results showed that the epithelial markers E-cadherin and a-catenin were upregulated, whereas the levels of the mesenchymal markers $\mathrm{N}$ cadherin, fibronectin, and vimentin were decreased when SH3BP5-AS1 was silenced (Fig. 2E). The opposite results were obtained when SH3BP5-AS1 was overexpressed in PC cells (Fig. S3C). Stem celllike cell markers Lin28, OCT4, NANOG, and SOX-2 have been found to be related to cell stemness [12], and these proteins were significantly downregulated when SH3BP5-AS1 was silenced and upregulated when SH3BP5-AS1 was overexpressed (Fig. 2J and K, Fig. S3F and G). These findings suggested that SH3BP5AS1 stimulates PC cell migration, invasiveness, and stemness.

\section{SH3BP5-AS1 promotes pancreatic cancer cell resistance to gemcitabine in vitro and in vivo}

These above results strongly supported the notion that SH3BP5-AS1 is involved in PC carcinogenicity; however, whether SH3BP5-AS1 affects GEM chemoresistance in PC still remained to be elucidated. First, lentiviral vectors encoding SH3BP5-AS1 inserts or shRNA were transfected into BxPC3 and MIA Paca-2 cells. Then the cells were treated with GEM or DMSO for $72 \mathrm{~h}$. We found that the PC cell proliferation ability was significantly reduced when SH3BP5-AS1 was silenced compared to the negative control group 
(Fig. 3A and B, Fig. S3A and B). Moreover, SH3BP5-AS1 silencing enhanced the inhibitory effect of GEM on PC cells (Fig. S3E and F). To corroborate the above results, cell viability was tested after exposing the cells to different GEM concentrations for $48 \mathrm{~h}$. The results showed that silencing of SH3BP5-AS1 enhanced the sensitivity of PC cells to GEM. Compared with the control group, the $\mathrm{IC}_{50}$ value of GEM was significantly decreased in the SH3BP5-AS1 silencing group (Fig. 3C, Fig. S3C). On the contrary, after overexpression of SH3BP5-AS1, the sensitivity of the PC cell line PANC-1 to GEM was significantly reduced (Fig. S3E-H). Furthermore, we found that silencing of SH3BP5-AS1 reduced the viability of GEMtreated PC cells in a time-dependent manner (Fig. 3D, Fig. S3D).

We further explored the effects of SH3BP5-AS1 on the sensitivity of PC to GEM through in vivo experiments. Stably silenced SH3BP5-AS1 PC cell lines and control cell lines were injected subcutaneously and via the tail vein, and the mice received $50 \mathrm{mg} / \mathrm{kg}$ GEM or saline. After 4 weeks, a significantly lower tumor volume and weight were observed in the SH3BP5-AS1 silencing group, after treatment with either GEM or saline, especially in the GEM treatment group (Fig. 3E-G). The Ki67 immunostaining results suggested that silencing of SH3BP5-AS1 inhibits proliferation and enhances GEM sensitivity of PC cells (Fig. $3 \mathrm{H}$ ). In the lung metastasis model, we found that silencing of SH3BP5AS1 significantly reduced the distant metastasis of the tumor (7/16) compared with the control group (13/16). Interestingly, compared with the GEM treatment group, silencing of SH3BP5-AS1 can significantly improve the efficacy of GEM, which reduced the lung metastasis rate and the number of lung metastases (Fig. $3 \mathrm{I}$ and $\mathrm{J}$ ). The above results suggested that SH3BP5-AS1 silencing enhanced the sensitivity of PC cells to GEM both in vitro and in vivo.

\section{ALKBH5/IGF2BP1-mediated m6A modification contributed to SH3BP5-AS1 overexpression}

m6A modification has been confirmed to be involved in the regulation of RNA expression[13]. m6A modifications are placed and removed by methylases and demethylases, respectively. ALKBH5, a demethylase, is well known to be involved in the occurrence and development of various cancers [14-16]. In this study, we found that ALKBH5 was significantly downregulated in tumor tissues compared to the adjacent tissues (Fig. 4A), and its expression was negatively correlated with SH3BP5-AS1 levels in tumor tissues (Fig. 4B). m6A RIP results showed that m6A levels in PC cell lines (BxPC-3 and PANC-1) were significantly higher than in normal pancreatic cells (HPDE6-C) (Fig. 4C). The in vitro results also revealed that ALKBH5 was significantly downregulated while SH3BP5-AS1 was overexpressed; on the contrary, ALKBH5 upregulation was observed after SH3BP5-AS1 was silenced (Fig. 4D and E). The dual luciferase report assay indicated that ALKBH5 could attenuate luciferase activity in the wild-type SH3BP5-AS1 group; however, luciferase activity was not affected in the mutant SH3BP5-AS1 group (Fig. 4F). MeRIP assays showed that ALKBH5 silencing increased the m6A modification level of SH3BP5-AS1 (Fig. 4G), whereas overexpression of ALKBH5 decreased the m6A modification level of SH3BP5-AS1 in PC cells (Fig. 4H). The actinomycin D assay (a drug that blocks de novo RNA synthesis) showed that ALKBH5 promoted SH3BPH5-AS1 degradation, while silencing of ALKBH5 contributed to the stability of SH3BP5AS1 (Fig. 4I). Collectively, ALKBH5 acted as an important "eraser" in regulating SH3BP5-AS1. However, an "eraser" cannot regulate SH3BP5-AS1 expression alone; it also requires a "reader." Studies have found 
that ALKBH5-mediated m6A demethylation inhibited mRNA transcription by the m6A effector IGF2BP1 $[15,17]$. Our correlation analysis revealed that IGF2BP1 expression was positively correlated with SH3BP5-AS1 levels (Fig. 4J). We hypothesized that IGF2BP1 acted as a "reader" for the regulation of SH3BP5-AS1. To confirm the above hypothesis, the RIP assay was performed to affirm the direct binding between IGF2BP1 and SH3BP5-AS1 (Fig. 4K). SH3BP5-AS1 expression was significantly decreased after IGF2BP1 silencing and increased after IGF2BP1 overexpression (Fig. $4 \mathrm{~L}$ and M). The results of our actinomycin $\mathrm{D}$ assay confirmed the above conclusion (Fig. $4 \mathrm{~N}$ ). Then an RIP assay was conducted to explore the correlation between the "eraser" ALKBH5 and the "reader" IGF2BP1. The results suggested that IGF2BP1 expression was negatively correlated with ALKBH5 levels (Fig. 40). In conclusion, low expression of the "eraser" ALKBH5 maintains a high m6A modification level of SH3BP5-AS1, which further promotes the recognition of m6A-SH3BP5-AS1 by the "reader" IGF2BP1.

\section{miR-139-5p is involved in the oncogenic roles of SH3BP5-AS1 in PC}

It has been reported that IncRNAs exert carcinogenic effects through acting as ceRNAs [18-20]. Here, we explored whether this is also the case for SH3BP5-AS1. First, Combined with the public database ENCORI, previously known as starBase v2.0, and the DIANA Tool, miRNA-194-5p, miRNA-589-5p, miRNA-26a-5p and miRNA-139-5p were predicted to be a target miRNA of SH3BP5-AS1 (Fig. 5A). Furthermore, RNA pulldown assay was performed to prove miRNA-139-5p was the most enriched in the precipitate of SH3BP5-AS1 than other miRNA candidates (Fig. 5B). Therefore, miRNA-139-5p was selected for follow-up study. RT-PCR was performed to further prove the interaction between miRNA-139-5p and SH3BP5-AS1. The results suggested that SH3BP5-AS1 expression is negatively correlated with miRNA-139-5p levels (Fig. 5C). The results of our luciferase assays confirmed that in both H293T and PC cells, miR139-5p significantly decreased the luciferase activity of wild-type SH3BP5-AS1 but did not affect that of mutant SH3BP5-AS1 (Fig. 5D-F). Furthermore, silencing of SH3BP5-AS1 can significantly upregulate the expression of miR139-5p, whereas the opposite results were obtained after SH3BP5-AS1 overexpression (Fig. 5G and K). The above results indicated that SH3BP5-AS1 might sponge miR139-5p, thereby affecting downstream target genes. The relationship was verified by RIP assay. We found that both SH3BP5-AS1 and miR139-5p were enriched in AGO2 precipitate (Fig. $5 \mathrm{H}-\mathrm{J}, \mathrm{L}-\mathrm{N}$ ). The results of our RNA pull-down assays confirmed the above results. miR-139-5p and AGO2 were more strongly enriched in the biotin-labeled SH3BP5-AS1 group than in the control group (Fig. 50-R). These results indicated that SH3BP5-AS1/miR-139-5P participate in the carcinogenic mechanism of PC.

To further explore the effect of SH3BP5-AS1/miRNA-139-5p on the biological behavior of PC and GEM resistance, first of all, Transwell and colony formation assays proved that silencing of SH3BP5-AS1 could reduce the invasion, migration, and spheroidizing abilities of PC cells, while miRNA-139-5p inhibition could reverse the effect of shSH3BP5-AS1 on PC cells. On the contrary, overexpression of miRNA-139-5p could reverse the carcinogenic effects of SH3BP5-AS1 (Fig. S4A-I). In the drug resistance intervention experiment, we found that miRNA-139-5p inhibition could reverse the effect of shSH3BP5-AS1 on GEM 
sensitivity of PC cells. Overexpression of miRNA-139-5p increased the sensitivity of PC cells to GEM. This further illustrates that SH3BP5-AS1 can affect the biological behavior and GEM resistance of PC cells through miRNA-139-5p (Fig. S4J-0).

\section{miR-139-5p sponging activity decreases CTBP1 levels, affecting the biological behavior of PC cells}

As shown above, miR-139-5p could inhibit the expression of downstream target genes by forming a RISC complex with AG02. Transcriptome sequencing was used to further screen downstream target genes. As shown in Fig. 5A and B, we found that CTBP1 was significantly downregulated when miR-139-5P was overexpressed. We conducted functional enrichment and signal pathway analyses of differentially expressed genes and found that genes, including CTBP1, are mainly enriched in Wnt signaling (Fig. 5C and D). Based on the miR-TARGET database to predict miRNA target genes, it was found that there were a total of 218 genes that could be regulated by miR-139-5p. Among the overlapping genes, the difference in CTBP1 expression is particularly obvious (Fig. 5C-E), indicating that CTBP1 might be a hub gene downstream of miR-139-5p. To prove this hypothesis, first, correlation analysis was conducted, and it was found that the expression of miR-139-5p was negatively correlated with CTBP1 levels (Fig. 6A). Then a luciferase assay confirmed that miRNA-139-5p could inhibit the fluorescence activity of wild-type CTBP1 without affecting the fluorescence activity of mutant CTBP1, confirming the regulatory relationship between miRNA-139-5p and CTBP1 (Fig. 6B and C). Overexpression of miR-139-5p downregulated CTBP1 mRNA and protein expression levels, while silencing of miR-139-5p upregulated CTBP1 mRNA and protein expression levels (Fig. 6D-F). All these results indicated that miR-139-5p has a targeted regulatory relationship with CTBP1. In the drug resistance study, we co-transfected miR-139-5p mimics and CTBP1 overexpression plasmid into PC cells, which were subsequently treated with GEM. We found that CTBP1 could reverse the effects of miR-139-5p on PC cell invasion, migration, and GEM resistance (Fig. 7A-I). On the contrary, when miR-139-5p and CTBP1 were silenced at the same time, si-CTBP1 could significantly inhibit PC cell invasion and migration, stemness, and GEM resistance (Fig. 7J-0). Therefore, CTBP1 is an important molecule downstream of miR-139-5p, and miR-139-5p exerts tumorigenic effects by regulating CTBP1.

\section{SH3BP5-AS1 localization contributes to the effects of CTBP1 and increases tumor malignancy via the Wnt/ $\beta$-catenin pathway}

SH3BP5-AS1 participates in the GEM resistance of PC cells through a ceRNA mechanism, as confirmed in the above described experiments. We detected the expression and location of SH3BP5-AS1 by RT-PCR and immunofluorescence, and found that SH3BP5-AS1 is expressed in both the nucleus and the cytoplasm (Fig. 6). We hypothesized that SH3BP5-AS1 might be transferred to the cytoplasm after regulation in the nucleus. When we further analyzed the regulatory relationship between SH3BP5-AS1 and CTBP1, we found that SH3BP5-AS1 silencing reduced cell invasion, migration, and stemness and 
enhanced GEM sensitivity in PC cells, whereas CTBP1 could reverse the abovementioned effects of shSH3BP5-AS1 (Fig. 8A and B, Fig. 7A and C). Conversely, silencing of CTBP1 could significantly inhibit the biological effects of SH3BP5-AS1 overexpression on PC cells (Fig. 7B and D). Similarly, CTBP1 restored the effects of sh-SH3BP5-AS1 on GEM sensitivity in PC cells, as observed in the drug sensitivity assay (Fig. 8D and E). Further correlation analysis revealed that the expression of SH3BP5-AS1 was negatively correlated with CTBP1 levels (Fig. 8F).

According to our transcriptome sequencing results, the Wnt pathway might be an important signaling pathway for SH3BP5-AS1/miRNA-139-5p/CTBP1 to exert its effect. We further detected Wnt pathwayrelated proteins through western blot and IHC and found that after SH3BP5-AS1 overexpression, the Wnt pathway was significantly activated (Fig. 8G and H). When CTBP1 was silenced at the same time, the Wnt signaling pathway was blocked. These results indicated that SH3BP5-AS1/miRNA-139-5p/CTBP1 might exert their biological functions via the Wnt signaling pathway.

\section{Discussion}

Chemoresistance has always been a frequently encountered bottleneck in the treatment of PC. Many studies on the effects of IncRNA on tumor chemoresistance and the underlying mechanisms have been conducted, aiming to find a marker with good specificity and sensitivity $[6,21,22]$. In this study, a PDX model combined with high-throughput sequencing was used to screen the key IncRNAs in PC chemoresistance. SH3BP5-AS1 is closely related to PC drug resistance. Only two studies have reported that SH3BP5-AS1 might be related to inflammation and the prognosis of head and neck tumors [9, 23]; however, the role of SH3BP5-AS1 has not been studied in PC. It is important to fully elucidate the mechanism of SH3BP5-AS1 in the drug resistance of PC. In this study, we found that high SH3BP5-AS1 expression promoted the invasion, migration, and stemness of PC cells and increased the resistance of PC cells to GEM. Furthermore, this study also revealed that SH3BP5-AS1 activates the Wnt signaling pathway through a ceRNA mechanism, which affects carcinogenesis and chemoresistance in PC.

More and more studies have shown that IncRNAs play important roles in the occurrence and development of malignant tumors including PC [6, 21, 24], This study found that the expression of SH3BP5-AS1 in PC (i) is significantly higher than that in normal pancreatic tissue and (ii) plays a role in promoting tumorigenesis. The results were also consistent with previous reports. For example, it has been reported that SH3BP5-AS1 (i) promotes the occurrence and development of head and neck cancer and (ii) is an independent risk factor for its prognosis [9]. In this study, correlation analysis of the clinicopathological characteristics and SH3BP5-AS1 expression suggested that high SH3BP5-AS1 expression is closely related to the TNM stage and poor prognosis of PC. This evidence proves the important role of SH3BP5AS1 in PC. Moreover, we also found that SH3BP5-AS1 overexpression might be regulated by m6A modification. m6A modification has been confirmed to be involved in the regulation of gene stability. It participates in the occurrence of a variety of tumors by regulating RNA transcription, splicing, processing, translation, and degradation [25]. m6A modifications are established and removed by $\mathrm{m} 6 \mathrm{~A}$ methyltransferases (writers) or demethylases (erasers), respectively, and recognized by m6A-binding 
proteins (readers), which ultimately affects the expression levels of RNAs. There are many studies on the roles of m6A modification in PC. The results of this study suggested that the "eraser" ALKBH5 and the "reader" IGF2BP1 are involved in the regulation of SH3BP5-AS1 expression. ALKBH5 has been confirmed to be downregulated in PC, and low ALKBH5 expression promotes the occurrence and development of tumors $[16,26]$. The clinical analyses of our center are consistent with previous reports. ALKBH5 expression was negatively correlated with SH3BP5-AS1 levels. Subsequent results confirmed that low ALKBH5 expression increased the level of SH3BP5-AS1 m6A modification, which is further recognized by the m6A recognition protein IGF2BP1, thereby promoting the expression of SH3BP5-AS1. This result also explains why SH3BP5-AS1 was highly expressed in PC. This is the first report of the regulatory mechanism of m6A modification of SH3BP5-AS1 in PC.

In this study, a new IncRNA, SH3BP5-AS1, affects GEM resistance in PC was discovered. A lack of SH3BP5-AS1 expression could enhance the sensitivity of PC cells to GEM. In vivo and in vitro experiments confirmed that SH3BP5-AS1 was involved in GEM resistance of PC. We found that SH3BP5-AS1 was expressed simultaneously in the nucleus and cytoplasm. We hypothesized that it might exert different functions in different subcellular compartments. Our previous study confirmed that IncRNAs might exert their effects via both nuclear and cytoplasmic pathways [27]. Our experiments have proved that SH3BP5AS1 is modified by m6A in the nucleus, leading to its high expression, and then part of SH3BP5-AS1 is transferred to the cytoplasm, further regulating miR-139-5p expression by the sponge effect. Our previous studies have confirmed that low expression of miR-139-5p promotes the occurrence and development of $\mathrm{PC}$, and miR-139-5p has also been confirmed to participate in the drug resistance of tumors including non-small cell lung cancer, ovarian cancer, and colon cancer[21]. CTBP1, a downstream target gene of miR-139-5p, was screened by transcriptome sequencing in this study. CTBP1 has been reported to be involved in tumor chemoresistance [28]; its high expression led to the occurrence of EMT and promoted the invasion and migration of tumor cells[29]. The results of this study are consistent with the conclusions of the above studies. Our study revealed that high SH3BP5-AS1 expression further upregulated the expression of CTBP1 through downregulation of miR-139-5p, which ultimately led to GEM resistance in PC. However, whether SH3BP5-AS1 participates in other regulatory mechanisms in the nucleus has not been explored. Moreover, it remains to be elucidated whether SH3BP5-AS1 can directly regulate the expression of the downstream target gene CTBP1 and how SH3BP5-AS1 exerts its regulatory effects.

To explore the mechanisms by which the SH3BP5-AS1/miR-139-5p/CTBP1 axis affects GEM resistance in PC cells, high-throughput sequencing and signal pathway enrichment analysis were conducted. We found that when SH3BP5-AS1 competitively bound miR-139-5p via ceRNA to regulate the downstream gene $C T B P 1$, it further regulated the Wnt signaling pathway. The Wnt signaling pathway has been confirmed to be involved in the tumorigenesis and drug resistance of a variety of tumors, including PC [21]. Studies have found that activation of the Wnt signaling pathway promotes the resistance of PC to GEM and the aggressiveness of tumors. 
CTBP1 is an essential molecule in the Wnt signaling pathway, which could affect cell proliferation and cell survival [30, 31]. The above results partially explain how abnormal SH3BP5-AS1 expression affects GEM resistance in PC cells and reveal its downstream regulatory mechanism. SH3BP5-AS1 may serve as a treatment target to enhance the sensitivity of PC to GEM.

\section{Conclusions}

This study revealed that SH3BP5-AS1 activated Wnt signaling pathway by sponging miR-139-5p, upregulating CTBP1 expression, and contributing to the sensitivity of PC cells to GEM. SH3BP5-AS1 is expected to a potential target for PC therapy.

\section{Abbreviations}

C-terminal binding protein 1, CTBP1; N6-methyladenosine, m6A; Pancreatic cancer, PC; Gemcitabine, GEM; Fetal bovine serum, FBS; Immunohistochemistry , IHC; Small interfering RNA, siRNA; RNA immunoprecipitation, RIP; American Type Culture Collection , ATCC; Dulbecco's modified Eagle's medium, DMEM; Short hairpin RNA, shRNA; Epithelial-to-mesenchymal transition, EMT.

\section{Declarations}

\section{Ethics approval and consent to participate}

The current study design and protocols were approved by the Ethics Committee of the First Affiliated Hospital of Fujian Medical University, China.

\section{Consent for publication}

All authors have agreed to publish this manuscript in Journal of Experimental \& Clinical Cancer Research.

\section{Availability of data and materials}

All the data obtained in current study were available from the corresponding authors on reasonable request.

\section{Competing interests}

The authors declare no competing interests.

\section{Funding}

This study was supported in part by the grants from Startup Fund for Scientific Reseach of Fujian Medical University (2019QH1064), the Natural Science Foundation of Fujian Province (2020J5248)》 Youth Scientific Reseach Project of Fujian Province Health Commission (2020QNA052). 


\section{Authors' contributions}

Chengjie Lin, Yan Wang and Yihong Dong carried out experiments and drafted the manuscript. Shihui Lai and Liang Wang collected the clinical samples and analyzed the data. Shangeng Weng and Xiang Zhang designed and directed the study. All the authors read and approved the final manuscript.

\section{Acknowledgments}

We thank LetPub (www.letpub.com) for its linguistic assistance during the preparation of this manuscript. We thank Ruikang Biotechnology Co., Ltd (Fujan, China) to provide products and technical support.

\section{References}

1. Siegel RL, Miller KD, Fuchs HE, Jemal A. Cancer Statistics, 2021. Cancer J Clin. 2021;71:7-33.

2. Rawla P, Sunkara T, Gaduputi V. Epidemiology of Pancreatic Cancer: Global Trends, Etiology and Risk Factors. World J Oncol. 2019;10:10-27.

3. Tempero M, Oh DY, Tabernero J, Reni M, Van Cutsem E, Hendifar A, et al. Ibrutinib in combination with nab-paclitaxel and gemcitabine for first-line treatment of patients with metastatic pancreatic adenocarcinoma: phase III RESOLVE study. Annals of oncology: official journal of the European Society for Medical Oncology. 2021;32:600-8.

4. Yang G, Guan W, Cao Z, Guo W, Xiong G, Zhao F, et al. Integrative Genomic Analysis of Gemcitabine Resistance in Pancreatic Cancer by Patient-derived Xenograft Models. Clinical cancer research: an official journal of the American Association for Cancer Research. 2021;27:3383-96.

5. Zhou C, Yi C, Yi Y, Qin W, Yan Y, Dong X, et al. LncRNA PVT1 promotes gemcitabine resistance of pancreatic cancer via activating Wnt/ $\beta$-catenin and autophagy pathway through modulating the miR619-5p/Pygo2 and miR-619-5p/ATG14 axes. Mol Cancer. 2020;19:118.

6. Xiong G, Liu C, Yang G, Feng M, Xu J, Zhao F, et al. Long noncoding RNA GSTM3TV2 upregulates LAT2 and OLR1 by competitively sponging let-7 to promote gemcitabine resistance in pancreatic cancer. J Hematol Oncol. 2019;12:97.

7. Shi W, Zhang C, Ning Z, Hua Y, Li Y, Chen L, et al. Long non-coding RNA LINC00346 promotes pancreatic cancer growth and gemcitabine resistance by sponging miR-188-3p to derepress BRD 4 expression. Journal of experimental clinical cancer research: CR. 2019;38:60.

8. Utnes P, Løkke C, Flægstad T, Einvik C. Clinically Relevant Biomarker Discovery in High-Risk Recurrent Neuroblastoma. Cancer informatics. 2019;18:1176935119832910.

9. Lina S. Identification of hub IncRNAs in head and neck cancer based on weighted gene co-expression network analysis and experiments. FEBS open bio. 2021;11:2060-73.

10. Wang T, Kong S, Tao M, Ju S. The potential role of RNA N6-methyladenosine in Cancer progression. Mol Cancer. 2020;19:88. 
11. Song H, Liu D, Dong S, Zeng L, Wu Z, Zhao P, et al. Epitranscriptomics and epiproteomics in cancer drug resistance: therapeutic implications. Signal transduction targeted therapy. 2020;5:193.

12. Heo JS, Choi Y, Kim HS, Kim HO. Comparison of molecular profiles of human mesenchymal stem cells derived from bone marrow, umbilical cord blood, placenta and adipose tissue. Int $\mathrm{J}$ Mol Med. 2016;37:115-25.

13. Zhang T, Zhang SW, Zhang SY, Gao SJ, Chen Y, Huang Y. m6A-express: uncovering complex and condition-specific m6A regulation of gene expression. Nucleic acids research 2021.

14. Jiang X, Liu B, Nie Z, Duan L, Xiong Q, Jin Z, et al. The role of m6A modification in the biological functions and diseases. Signal transduction targeted therapy. 2021;6:74.

15. Chen Y, Zhao Y, Chen J, Peng C, Zhang Y, Tong R, et al. ALKBH5 suppresses malignancy of hepatocellular carcinoma via mA-guided epigenetic inhibition of LYPD1. Mol Cancer. 2020;19:123.

16. Guo X, Li K, Jiang W, Hu Y, Xiao W, Huang Y, et al. RNA demethylase ALKBH5 prevents pancreatic cancer progression by posttranscriptional activation of PER1 in an m6A-YTHDF2-dependent manner. Mol Cancer. 2020;19:91.

17. Zhang X, Zhong L, Zou Z, Liang G, Tang Z, Li K, et al. Clinical and Prognostic Pan-Cancer Analysis of N6-Methyladenosine Regulators in Two Types of Hematological Malignancies: A Retrospective Study Based on TCGA and GTEx Databases. Frontiers in oncology. 2021;11:623170.

18. Li SY, Zhu Y, Li RN, Huang JH, You K, Yuan YF, et al: aLncRNA Lnc-APUE is Repressed by HNF4 and Promotes G1/S Phase Transition and Tumor Growth by Regulating MiR-20b/E2F1 Axis. Advanced science (Weinheim, Baden-Wurttemberg, Germany) 2021, 8:2003094.

19. Sun CC, Zhu W, Li SJ, Hu W, Zhang J, Zhuo Y, et al. FOXC1-mediated LINC00301 facilitates tumor progression and triggers an immune-suppressing microenvironment in non-small cell lung cancer by regulating the HIF1 a pathway. Genome medicine. 2020;12:77.

20. Wang W, Hu W, Wang Y, An Y, Song L, Shang P, et al. Long non-coding RNA UCA1 promotes malignant phenotypes of renal cancer cells by modulating the miR-182-5p/DLL4 axis as a ceRNA. Mol Cancer. 2020;19:18.

21. Huang N, Guo W, Ren K, Li W, Jiang Y, Sun J, et al. LncRNA AFAP1-AS1 Supresses miR-139-5p and Promotes Cell Proliferation and Chemotherapy Resistance of Non-small Cell Lung Cancer by Competitively Upregulating RRM2. Frontiers in oncology. 2019;9:1103.

22. Yang Q, Li K, Huang X, Zhao C, Mei Y, Li X, et al. IncRNA SLC7A11-AS1 Promotes Chemoresistance by Blocking SCF-Mediated Degradation of NRF2 in Pancreatic Cancer. Molecular therapy Nucleic acids. 2020;19:974-85.

23. Lu JW, Rouzigu A, Teng LH, Liu WL. The Construction and Comprehensive Analysis of InflammationRelated ceRNA Networks and Tissue-Infiltrating Immune Cells in Ulcerative Progression. BioMed research international. 2021;2021:6633442.

24. Su SC, Yeh CM, Lin CW, Hsieh YH, Chuang CY, Tang $\mathrm{CH}$, et al: A novel melatonin-regulated IncRNA suppresses TPA-induced oral cancer cell motility through replenishing PRUNE2 expression. Journal of pineal research 2021:e12760. 
25. Zheng J, Zhao Z, Wan J, Guo M, Wang Y, Yang Z, et al: N-6 methylation-related IncRNA is potential signature in lung adenocarcinoma and influences tumor microenvironment. Journal of clinical laboratory analysis 2021:e23951.

26. Tang $B$, Yang $Y$, Kang $M$, Wang $Y$, Wang $Y, B i ~ Y$, et al. mA demethylase ALKBH5 inhibits pancreatic cancer tumorigenesis by decreasing WIF-1 RNA methylation and mediating Wnt signaling. Mol Cancer. 2020;19:3.

27. Jia G, Wang Y, Lin C, Lai S, Dai H, Wang Z, et al. LNCAROD enhances hepatocellular carcinoma malignancy by activating glycolysis through induction of pyruvate kinase isoform PKM2. Journal of experimental clinical cancer research: CR. 2021;40:299.

28. Paredes R, Schneider M, Stevens A, White DJ, Williamson AJK, Muter J, et al. EVI1 carboxy-terminal phosphorylation is ATM-mediated and sustains transcriptional modulation and self-renewal via enhanced CtBP1 association. Nucleic acids research. 2018;46:7662-74.

29. Sahu SK, Tiwari N, Pataskar A, Zhuang Y, Borisova M, Diken M, et al. FBXO32 promotes microenvironment underlying epithelial-mesenchymal transition via CtBP1 during tumour metastasis and brain development. Nature communications. 2017;8:1523.

30. Huard CC, Tremblay CS, Helsper K, Delisle MC, Schindler D, Lévesque G, et al. Fanconi anemia proteins interact with CtBP1 and modulate the expression of the Wnt antagonist Dickkopf-1. Blood. 2013;121:1729-39.

31. Valenta T, Lukas J, Korinek V. HMG box transcription factor TCF-4's interaction with CtBP1 controls the expression of the Wnt target Axin2/Conductin in human embryonic kidney cells. Nucleic acids research. 2003;31:2369-80.

\section{Figures}

\section{Figure 1}

\section{SH3BP5-AS1 was upregulated in GEM-treated PDX pancreatic cancer and its upregulation was negatively correlated with overall survival.}

(A) Establishment of the PDX pancreatic cancer model and experimental workflow of GEM treatment. (B) Heatmap displaying the top 10 upregulated IncRNAs in GEM-treated and control PDX mice. (C) Correlation analysis of SH3BP5-AS1 and clinicopathological features based on the TCGA database.

(D) SH3BP5-AS1 was upregulated in pancreatic cancer tumor tissues compared with the paired adjacent tissues, as revealed by RT-qPCR. (E) SH3BP5-AS1 is an independent risk factor for the prognosis of pancreatic cancer, as revealed by ROC curve analysis. (F) Upregulation of SH3BP5-AS1 was correlated with poor overall survival. (G) SH3BP5-AS1 expression was significantly higher in pancreatic cancer cells compared with HPDE6-C7 cells. Data are shown as the mean \pm SD. ${ }^{\star} P<0.05 ; \star \star P<0.01$. 


\section{Figure 2}

Silencing of SH3BP5-AS1 expression inhibited the invasion, migration, and stemness of pancreatic cancer cells in vitro.

vivo. (A, B) Transwell assay revealed the migration and invasion abilities of BxPC-3 and MIA Paca-2 cells transfected with shCtr or shSH3BP5-AS1. (C, D) Histogram displaying the statistical results of the Transwell assay. (E) EMT-related markers were detected by western blot. (F-I) Colony formation analysis. $(\mathrm{J}-\mathrm{L})$ Stem cell-like cell markers Lin28, OCT4, NANOG, and SOX-2 were detected by western blot. Data are shown as the mean \pm SD of three replicates. ${ }^{\star} P<0.05 ;{ }^{\star} * P<0.01$.

\section{Figure 3}

Upregulation of SH3BP5-AS1 promotes pancreatic cancer cell resistance to gemcitabine in vitro and in vivo.

(A, B) Colony formation in BXPC-3 cells transfected with shCtr or shSH3BP5-AS1 and treated with DMSO or GEM. (C) BxPC-3 cells with stable silencing of SH3BP5-AS1 were treated with GEM at different concentrations for $48 \mathrm{~h}$. Cell viability was measured by MTT assay. (D) Stable SH3BP5-AS1-silenced BxPC-3 cells were treated with GEM $(1 \mu \mathrm{M})$ for $48 \mathrm{~h}$. Cell viability was measured by MTT assay at different timepoints. (E) Representative images of the xenograft tumors of BxPC-3 cells with stable SH3BP5-AS1 silencing in nude mice following GEM or saline treatment. $(F, G)$ Histograms displaying the tumor weight and volume. $(\mathrm{H}) \mathrm{Ki67}$ expression in BxPC-3 cells with stable SH3BP5-AS1 silencing in nude mice after GEM or saline treatment. $(\mathrm{J})$ Table summarizing the results of lung metastasis. $(\mathrm{K})$ Representative images of H\&E staining of the xenograft tumors. Data are shown as the mean $\pm \mathrm{SD}$. ${ }^{\circ}<$ $0.05 ; * \star P<0.01$.

\section{Figure 4}

\section{ALKBH5/IGF2BP1-mediated m6A modification contributed to SH3BP5-AS1 overexpression.}

(A) ALKBH5 was downregulated in pancreatic cancer tumor tissues compared with the paired adjacent tissues, as revealed by RT-qPCR. (B) The negative correlation between the levels of ALKBH5 and SH3BP5AS1 was confirmed using the TCGA database. (C) The RIP RT-qPCR assay revealed that m6A modification of SH3BP5-AS1 was mainly enriched in pancreatic cancer cells (BXPC-3, PANC-1) compared with HPDE6-C7. (D, E) The expression level of ALKBH5 when SH3BP5-AS1 was overexpressed or silenced. (F) The relationship between ALKBH5 and SH3BP5-AS1 was confirmed by the luciferase reporter assay. 
$(G, H)$ RIP qRT-PCR results showing the m6A modification level of SH3BP5-AS1 when ALKBH5 was overexpressed or silenced. (I) SH3BP5-AS1 stability analysis in BXPC-3 cells with ALKBH5 overexpression or knockdown in the presence of actinomycin $D$. $(J)$ The negative correlation between the levels of ALKBH5 and IGF2BP1 in pancreatic cancer cells. (K) The enrichment of IGF2BP1 on SH3BP5-AS1 in pancreatic cancer cells was analyzed by RIP RT-qPCR assay. $(L, M)$ The expression level of SH3BP5-AS1 when IGF2BP1 was overexpressed or knocked down. $(\mathrm{N})$ Actinomycin $\mathrm{D}$ assays showing the effects of ALKBH5 overexpression or knockdown on IGF2BP1 stability. (0) RIP RT-qPCR assay results showing the enrichment of IGF2BP1 on SH3BP5-AS1 upon ALKBH5 silencing. Data are shown as the mean \pm SD. ${ }^{*} P<$ $0.05 ; * \star P<0.01$.

\section{Figure 5}

\section{miR-139-5p is involved in the oncogenic roles of SH3BP5-AS1 in pancreatic cancer.}

(A) Venn diagram showing the potential target miRNAs of SH3BP5-AS1 based on the public database ENCORI and the DIANA Tool. (B) RNA pulldown assay was performed to prove miRNA-139-5p was the most enriched in the precipitate of SH3BP5-AS1 than other miRNA candidates. (C) Correlation analysis of SH3BP5-AS1 and miRNA-139-5p. (D) Schematic diagram of the binding sites between SH3BP5-AS1 and miRNA-139-5p. (E, F) A dual luciferase reporter assay was conducted to determine the relative luciferase activities in pancreatic cancer cells following transfection with the indicated constructs. (G) miRNA-139$5 p$ was overexpressed following silencing of SH3BP5-AS1 in BxPC-3 and MIA Paca-2 cells. (H-J) An RIP assay was conducted to evaluate the relative enrichment of SH3BP5-AS1 and miRNA-139-5p in anti-lgGor anti-AG02-specific immunoprecipitates in H293T cells. (K) miRNA-139-5p was downregulated following SH3BP5-AS1 overexpression in PANC-1 cells. (L-N) An RIP assay was conducted to evaluate the relative enrichment of SH3BP5-AS1 and miRNA-139-5p in anti-IgG- or anti-AGO2-specific immunoprecipitates in MIA Paca-2 cells. (O-R) An RNA pull-down assay was conducted to detect the interaction among LNCAROD, miR-145-5p, and AGO2 in H293T and MIA Paca-2 cells. Data are shown as the mean \pm SD. ${ }^{*} P<0.05 ; * \star P<0.01$.

\section{Figure 6}

\section{miR-139-5p negatively regulated the expression of the target gene CTBP1.}

(A) Correlation analysis of CTBP1 and miRNA-139-5p. (B, C) A dual luciferase reporter assay was conducted to determine the relative luciferase activities following the indicated transfections in H293T and MIA Paca-2 cells. (D, E) CTBP1 was downregulated following miRNA-139-5p overexpression in BxPC3 and MIA Paca-2 cells and overexpressed following silencing of miRNA-139-5p in PANC-1 cells. $(F, G)$ 
CTBP1 protein expression was detected following the indicated transfections in BxPC-3, MIA Paca-2, and PANC-1 cells. Data are shown as the mean \pm SD. ${ }^{\star} P<0.05 ; * \star P<0.01$.

\section{Figure 7}

\section{miR-139-5p sponging activity decreases CTBP1 levels, affecting the biological behavior of pancreatic cancer.}

(A, B) Transwell and colony formation assays were performed in BxPC-3 cells after co-transfection with miRNA-139-5p mimics and/or CTBP1 overexpression plasmid. (C) Stem cell-like cell markers Lin28, OCT4, NANOG, and SOX-2 were detected by western blot following the indicated transfections in BxPC-3 cells. (D-E) Transwell and colony formation assays were performed in MIA Paca-2 cells following the indicated transfections. (F) Stem cell-like cell markers Lin28, OCT4, NANOG, and SOX-2 were detected by western blot following the indicated transfections in MIA Paca-2 cells. $(G, H)$ Transwell and colony formation assays were performed in PANC-1 cells after co-transfection with miRNA-139-5p-shRNA and CTBP1-shRNA. (I) Stem cell-like cell markers Lin28, OCT4, NANOG, and SOX-2 were detected by western blot following the indicated transfections in PANC-1 cells. (J-L) Pancreatic cancer cells with indicated transfections were treated with GEM at different concentrations for $48 \mathrm{~h}$. Cell viability was measured by MTT assay. $(M-0)$ Pancreatic cancer cells with indicated transfections were treated with GEM $(1 \mu M)$ for $48 \mathrm{~h}$. Cell viability was measured by MTT assay at different timepoints. Data are shown as the mean \pm SD. ${ }^{\star} P<0.05 ; * \star P<0.01$.

\section{Figure 8}

\section{SH3BP5-AS1 localization contributes to CTBP1 expression and increases tumor malignancy via the Wnt/ $\beta$-catenin pathway.}

(A-B) Transwell and colony formation assays were performed in BxPC-3 cells after co-transfection with SH3BP5-AS1-shRNA and/or CTBP1 overexpression plasmid. (C) Stem cell-like cell markers Lin28, OCT4, NANOG, and SOX-2 were detected by western blot following the indicated transfections in BxPC-3 cells. (D) BxPC-3 cells with indicated transfections were treated with GEM at different concentrations for $48 \mathrm{~h}$. Cell viability was measured by MTT assay. (E) BxPC-3 cells with indicated transfections were treated with GEM $(1 \mu \mathrm{M})$ for $48 \mathrm{~h}$. Cell viability was measured by MTT assay at different timepoints. (F) Correlation analysis of CTBP1 and miRNA-139-5p. (G) The proteins of Wnt/ $\beta$-catenin pathway were detected by western blot following the indicated transfections in BxPC-3 cells. $(H)$ Proteins of the Wnt/ $\beta$-catenin pathway were detected by IHC following the indicated transfections in BxPC-3 cells. Data are shown as the mean \pm SD. ${ }^{\star} P<0.05 ; * \star P<0.01$. 
Figure 9

Legend not included with this version

\section{Supplementary Files}

This is a list of supplementary files associated with this preprint. Click to download.

- Sfig1.tif

- Sfig2.tif

- Sfig3.tif

- Sfig4.tif

- Sfig5.tif

- Sfig6.tif

- Sfig7.tif

- Supplementaryfigurelegends.doc

- Supplementarytable1.doc

- Supplementarytable2.doc 